\title{
Die Insuffizienz der intraabdominellen Infektabwehr bei der eitrigen Peritonitis - Folge einer gestörten Fremdkörperopsonierung
}

\author{
A. Billing ${ }^{1}$, D. Fröhlich ${ }^{1}$, H. Kortmann ${ }^{1}$ und M. Jochum ${ }^{2}$ \\ ${ }^{1}$ Chirurgische Klinik und Poliklinik der Ludwig-Maximilians-Universität München, Klinikum Großhadern \\ ${ }^{2}$ Abteilung für Klinische Chemie und Klinische Biochemie in der Chirurgischen Klinik Innenstadt \\ der Ludwig-Maximilians-Universität München
}

\section{Deficient Intraabdominal Phagocytosis in Peritonitis Patients Secondary to Impaired Opsonization}

Summary. Despite a high concentration of serum proteins and intact phagocytes peritonitis exudates contain a large number of viable, pathogenic bacteria. The reason for this biological paradox is unknown. Our investigations reveal a pronounced defect in humoral opsonization of foreign particles in peritonitis exudate. We evaluated a modified chemiluminescence system allowing the determination of opsonic activity in serum and exudate. In serum we found a close correlation between opsonic activity and immunologically measurable levels of $\mathrm{C}_{3}$-complement and IgG. In purulent peritonitis exudates, however, the actual opsonizing activity was much less than expected according to the opsonin concentrations. We found a pronounced difference between immunologically determined opsonin levels and impaired opsonic function. Employing crossed immunoelectrophoresis massive $\mathrm{C}_{3}$-splitting into smaller fragments could be demonstrated in peritonitis exudates. In these exudates we found very high concentrations of granulocyte proteolytic (elastase) and oxidative (myeloperoxidase) enzymes which may lead to a functional destruction of opsonins followed by impaired opsonization in peritonitis exudate. The great number of bacteria and foreign particles in addition can cause a pronounced physiological consumption of complement components. The almost complete breakdown of intact $\mathrm{C}_{3}$-complement in intraabdominal exudate explains the deficient host defence in patients with severe peritonitis.

Key words: Peritonitis - Opsonization - Complement $-\mathrm{C}_{3}-\mathrm{IgG}-$ Granulocytic proteins

Abkürzungsverzeichnis: $\quad \mathrm{CL}=$ Chemilumineszenz; $\quad \operatorname{IgG}=\mathrm{Im}$ munglobulin $\mathrm{G} ; \mathrm{OK}=$ Opsonierungskapazität
Ein Versagen der Abwehrmechanismen am Peritonitisherd führt zu systemischen Komplikationen, wie Bakteriämie und Toxinämie mit konsekutiver Sepsis und Multiorganversagen. Charakteristisch für Peritonitisexsudate ist ein breites Spektrum vitaler, mikrobiologisch anzüchtbarer Bakterien trotz hoher Phagozytenzahlen (vorwiegend polymorphkernige (PMN-) Granulozyten). Da die Phagozytosefähigkeit, Hauptkomponente der zellulären Infektabwehr, in PMN-Granulozyten aus Peritonitisexsudaten erhalten bzw. sogar stimuliert ist (Billing 1986; Freischlag 1986), gibt es für die Diskrepanz zwischen Phagozytenzahl und Abwehrleistung bisher keine ausreichende Erklärung.

Über die Funktion der humoralen Abwehrfaktoren im menschlichen Peritonitisexsudat liegen keine gesicherten Erkenntnisse vor, lediglich eine Verminderung der IgG-Konzentration ist beschrieben (Duswald 1976; Eckert 1981). In der Frühphase der Erkrankung spielen die antigenunspezifischen Komponenten des Abwehrsystems die Hauptrolle (Hahn 1984; Stübner 1983). Die zentrale Funktion bei der Fremdkörpererkennung und Phagozytosevermittlung ist in der Aktivierung des Komplementsystems zu sehen. Diese führt zur Opsonierung des pathogenen Agens (Bakterien, Viren, Zelldetritus etc.), aber auch zur direkten Bakteriolyse und Leukotaxie. Das Verhalten von Opsonieringsfunktion und Opsoninspiegeln in der Zirkulation von Peritonitis- und Sepsispatienten gewann in den letzten Jahren zunehmendes Interesse (van Dijk 1982; Duswald 1976); vergleichbare Untersuchungen im Peritonitisexsudat fehlen. Die Anwendung modifizierter Chemilumineszenzverfahren ermöglicht im Serum die relativ einfache und verlä $B-$ liche Bestimmung der Opsonierungsfunktion (Allen 1977, 1984; Bellavite 1983). Ein entsprechendes Testsystem sollte für die Messung von Exsudatproben erstellt werden. 
Im eitrigen Exsudat ist mit einer hohen Konzentration granulozytärer Enzyme zu rechnen. Die Hemmung der Opsonierungsleistung durch solche Enzyme ist bekannt (Coble 1984), die Spaltung von $\mathrm{C}_{3}$-Komplement in Pleuraempyemflüssigkeit ist beschrieben (Waldvogel 1984). Über das Ausmaß der Freisetzung granulozytärer Enzyme in das eitrige Peritonitisexsudat und deren Auswirkung auf die intraperitoneale Opsonierung gibt es jedoch keine gesicherten Fakten.

\section{Material und Methoden}

Es wurden 21 Patienten mit diffus eitriger Peritonitis untersucht. Intraoperativ fand sich jeweils trübes Exsudat im gesamten Abdomen. In 8 Fällen wurde wegen einer klinisch mehr als $24 \mathrm{~h}$ alten, fibrinösen Peritonitis mit mehrfacher programmierter Abdominallavage (Etappenlavage) behandelt (Teichmann 1986). Peritonitisursache waren spontane gastrointestinale Perforationen sowie postoperativ entstandene Fisteln. $\mathrm{Zu} 28$ Operationszeitpunkten wurde Peritonitisexsudat intraoperativ mit einer Plastikspritze aspiriert. Das frische Exsudat wurde sofort für 10 min bei $2000 \mathrm{~g}$ zentrifugiert; im Überstand fanden sich danach keine zellulären Elemente mehr. Zu entsprechenden Zeitpunkten wurden Blutproben entnommen und zu Serum bzw. EDTA-Plasma verarbeitet. Bis zu ihrer Verwendung lagerten die Proben bei $-70^{\circ} \mathrm{C}$. Normale Serumproben wurden von gesunden Freiwilligen durch Venenpunktion gewonnen.

\section{Biochemische Untersuchungen}

\section{a) Bestimmung der Opsonierungsaktivität}

Die bei der Phagozytose aus Granulozyten pro Zelle freigesetzte Chemilumineszenz (CL) stellt, zumindest an normalen Zellen, ein Maß für die Pha- gozytoseleistung dar (Allen 1977). Letztere ist abhängig von der Aktivität der Phagozyten und von Art, Zahl und Opsonierung der phagozytierbaren Partikel. Werden in einem Testansatz alle Faktoren mit Ausnahme des opsonierenden Mediums konstant gehalten, kann mit einer CL-Messung Ausmaß und Qualität der Partikelopsonierung bestimmt werden.

Zymosan wurde mit den $\mathrm{zu}$ untersuchenden Proben (wahlweise Normalserum, Patientenserum, Peritonitisexsudat) voropsoniert: $1 \mathrm{ml}$ ZymosanLösung wurde mit $1 \mathrm{ml}$ der Probe für $15 \mathrm{~min}$ bei $37^{\circ} \mathrm{C}$ inkubiert. Nach dreimaligem Waschen wurde die Lösung in $0,9 \% \mathrm{NaCl}$ in einer Konzentration von $20 \mathrm{mg} / \mathrm{ml}$ resuspendiert. Jeder CL-Meßansatz enthielt $0,05 \mathrm{ml}$ verdünntes EDTA-Spenderblut ( $50 \mu \mathrm{l}$ in $700 \mu \mathrm{l}$ PBS), $0,8 \mathrm{ml}$ Veronal-Puffer und 0,1 ml Luminol-Lösung (Inthorn 1987). Die Vorinkubation des Ansatzes betrug $5 \mathrm{~min}$ bei $37^{\circ} \mathrm{C}$; die Reaktion wurde durch Zugabe von $0,05 \mathrm{ml}$ opsoniertem Zymosan gestartet. Die emittierte $\mathrm{CL}$ wurde bei $37^{\circ} \mathrm{C}$ mit einem 6-Kanal-Biolumat-Photometer (Fa. Berthold, Wildbad) gemessen. Der Verlauf der Photonenfreisetzung wurde mittels angekoppeltem Schreiber kontinuierlich aufgezeichnet und mit Hilfe eines Apple-2-Computers das Gesamt-CL-Integral über 30 min errechnet. Die Opsonierungskapazität wurde folgendermaßen ermittelt: In jedem Meßgang wurde jeweils parallel

1. mit Normalserum opsoniertes Zymosan

2. mit Patientenserum opsoniertes Zymosan und

3. mit Peritonitisexsudat opsoniertes Zymosan verwendet. Die resultierende CL als Maß für die Opsonierungskapazität wurde jeweils in einer Doppelbestimmung gemessen und der Mittelwert gebildet. Die CL, die durch das mit Normalserum opsonierte Zymosan erzeugt wurde, diente als $100 \%$ Referenzwert. Die CL- bzw. Opsonierungskapazität von Patientenserum bzw. Patientenexsudat

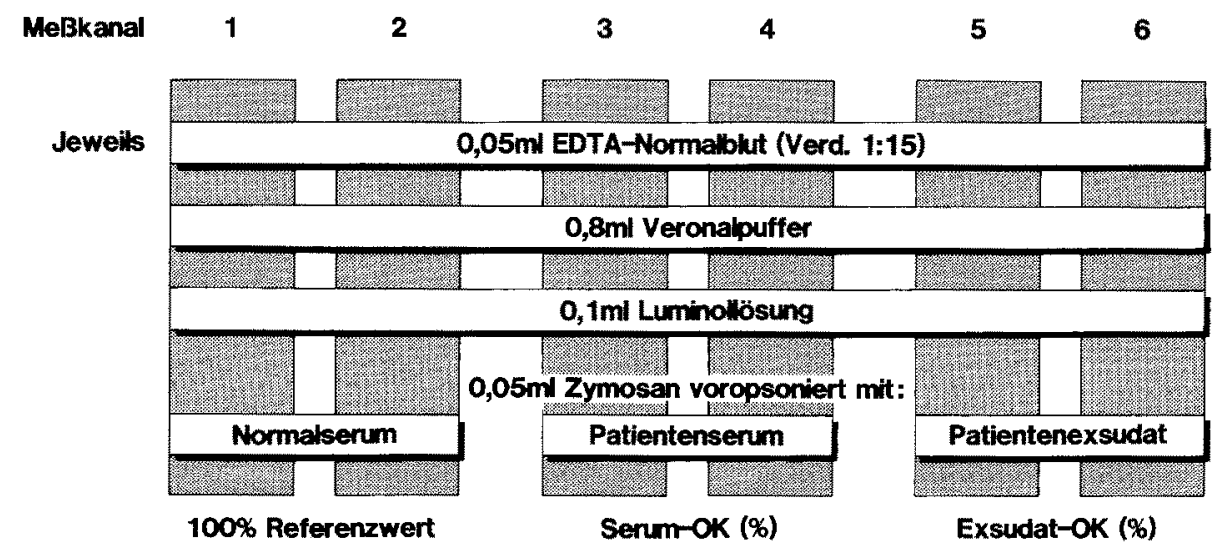

Abb. 1. Chemilumineszenzansatz zur Bestimmung der Opsonierungskapazität (OK) 
wurde in Relation zu diesem Wert gesetzt (s. Abb. 1).

b) Immunologische Messung der Opsonierungsfaktoren

Die quantitative Bestimmung von $\mathrm{C}_{3}$ und IgG erfolgte mittels standardisierter radialer Immundiffusion (NOR-Immundiffusionplatten, Behringwerke Marburg). GemäB den Angaben des Herstellers entsprechen dabei $1250 \mathrm{mg} / \mathrm{dl}$ IgG $100 \%$ und $82 \mathrm{mg} / \mathrm{dl} \mathrm{C}_{3} \quad 100 \%$ der Norm. Die Identifizierung von $\mathrm{C}_{3}$-Spaltprodukten erfolgte mit der zweidimensionalen Immunelektrophorese (Ganroth 1972). Es wurde ein gegen $C_{3 c}$ gerichteter, kommerzieller Antikörper (Behringwerke, Marburg) verwendet, der sowohl das intakte $\mathrm{C}_{3}$-Molekül, als auch $\mathrm{C}_{3 \mathrm{c}}$-haltige Fragmente erfaßt. Die Gel-Elektrophorese erfolgte in 1\% Agarose/TRIS-Barbiturat-Puffer $\left(\mathrm{pH} \quad 8,6\right.$ bei $10^{\circ} \mathrm{C}, 187 \mathrm{~V}, 12 \mathrm{~mA}$, Laufzeit $4 \mathrm{~h}$ ). Der Elektrophorese-Gelstreifen wurde dann in eine $\mathrm{C}_{3 \mathrm{c}}$-antikörperhaltige Agaroseplatte eingesetzt; die Elektrophorese in der 2. Dimension wurde unter den oben genannten Bedingungen durchgeführt.

\section{c) Bestimmung von Granulozytenproteinen}

Granulozytäre Elastase (im Komplex mit $\alpha_{1}$-Proteinaseinhibitor) wurde in Plasma und Exsudat mittels eines ELISAs der Fa. E. Merck, Darmstadt (PMN-Elastase Testkit) bestimmt (Neumann, 1984). Der Plasmanormbereich ist mit $50-181 \mu \mathrm{g} / 1$ angegeben. Granulozytäre Myeloperoxidase (Plasmanormbereich $25-47 \mu \mathrm{g} / 1$ ) wurde ebenfalls mittels ELISA gemessen (Neumann 1986).

\section{d) Leukozytenzahl und Proteinbestimmungen}

Leukozytenzählungen wurden in einer NeubauerZählkammer nach Färbung mit Türks-Lösung (Fa. Merck) durchgeführt. Die Proteinbestimmung erfolgte mit der Biuret-Methode (Weichselbaum 1946). Serum und Exsudat-Proteinelektrophoresen wurden nach (Grabner 1970) durchgeführt.

\section{Ergebnisse}

\section{Opsonierungsverhalten im Serum}

Die $\mathrm{C}_{3}$-Komplement-Spiegel im Serum (bezogen auf das Kontrollserum) lagen in diesem heterogenen Patientenkollektiv mit eitriger Peritonitis zwischen 27,1 und $105,0 \mathrm{mg} / \mathrm{dl}$ (das sind $33 \%$ und $128 \%$ des Serumnormalwertes), die IgG-Spiegel zwischen 162,5 und $2087,5 \mathrm{mg} / \mathrm{dl} \quad(13 \%$ und $167 \%$ ); die Opsonierungskapazität aller gemesse- nen Proben bewegte sich zwischen 26 und 172\% des Serumnormalwertes. Die Untergliederung der Proben in solche, die bei der Erstoperation wegen akuter Peritonitis und solche, die in der Folge bei Reoperationen (Etappenlavage) entnommen wurden, erlaubte eine Verlaufsbeobachtung. Während der Serumopsoninspiegel bei akut einsetzender Peritonitis an der unteren Normgrenze lagen, führte die länger anhaltende Infektion zu einer deutlichen Erhöhung, $\mathrm{C}_{3}$ stieg um durchschnittlich $27 \%$ und IgG um $74 \%$ an. Die Opsonierungskapazität zeigte ein gleichsinniges Verhalten und erhöhte sich um $35 \%$ (Tabelle 1). Die mit dem oben genannten Meßsystem bestimmte Zymosanopsonierung zeigte im Patientenserum eine deutliche Abhängigkeit von der Opsoninkonzentration. Bei Annahme eines linearen Zusammenhanges zwischen $\mathrm{C}_{3}$ bzw. IgG und der Opsonierungskapazität ergab sich jeweils eine Korrelation von $\mathrm{r}=0,75$ bzw. $\mathrm{r}=0,81$. Eine noch genauere Abschätzung der Opsonierungskapazität resultierte aus der Summe von $\mathrm{C}_{3^{-}}$ und IgG-Konzentrationen im Serum. Die Werte wurden hierfür in \% der Serumnorm eingesetzt und addiert, für Normalserum resultiert somit z.B. $200 \%$. Auf diese Weise wurde ein linearer Zusammenhang mit der Regressionsgeraden $y=34,97+$ $0,42 \times$ und einem Korrelationskoeffizient $r=0,82$ ermittelt.

Zur weiteren Klärung des Verhaltens der Opsonierungskapazität in Bereichen niedriger Opsoninkonzentrationen wurde eine Verdünnungsreihe von Normalserum auf die jeweilige Osponierungsleistung hin untersucht. Serumverdünnungen auf weniger als $10 \%$ zeigten eine niedrige Opsonierungs-Aktivität, es folgte dann ein relativ steiler Anstieg der Opsonierungsleistung bis zu einer Serumverdünnung von $25 \%$ und danach ein annähernd linearer, wenig steiler Kurvenverlauf (Abb. 2).

Die Verdünnungskurve des Normalserums wurde auf ihre Gültigkeit für das Serum der Peritonitispatienten untersucht. Es bestand kein wesentlicher Unterschied in der Abhängigkeit von Opsoninspiegel und Opsonierungskapazität zwischen Normal- und Patientenseren: Die Verdünnungskurve des Normalserums lag im Bereich der Punktewolke der Patientendaten (Abb. 2). Die immunologisch gemessenen Opsoninkonzentrationen erlaubten somit im Serum einen Rückschluß auf die funktionelle Opsonierungskapazität.

\section{Opsonierungsverhalten im Exsudat}

Eine wesentliche Voraussetzung für die Fremdkörperopsonierung durch IgG und Komplement am 


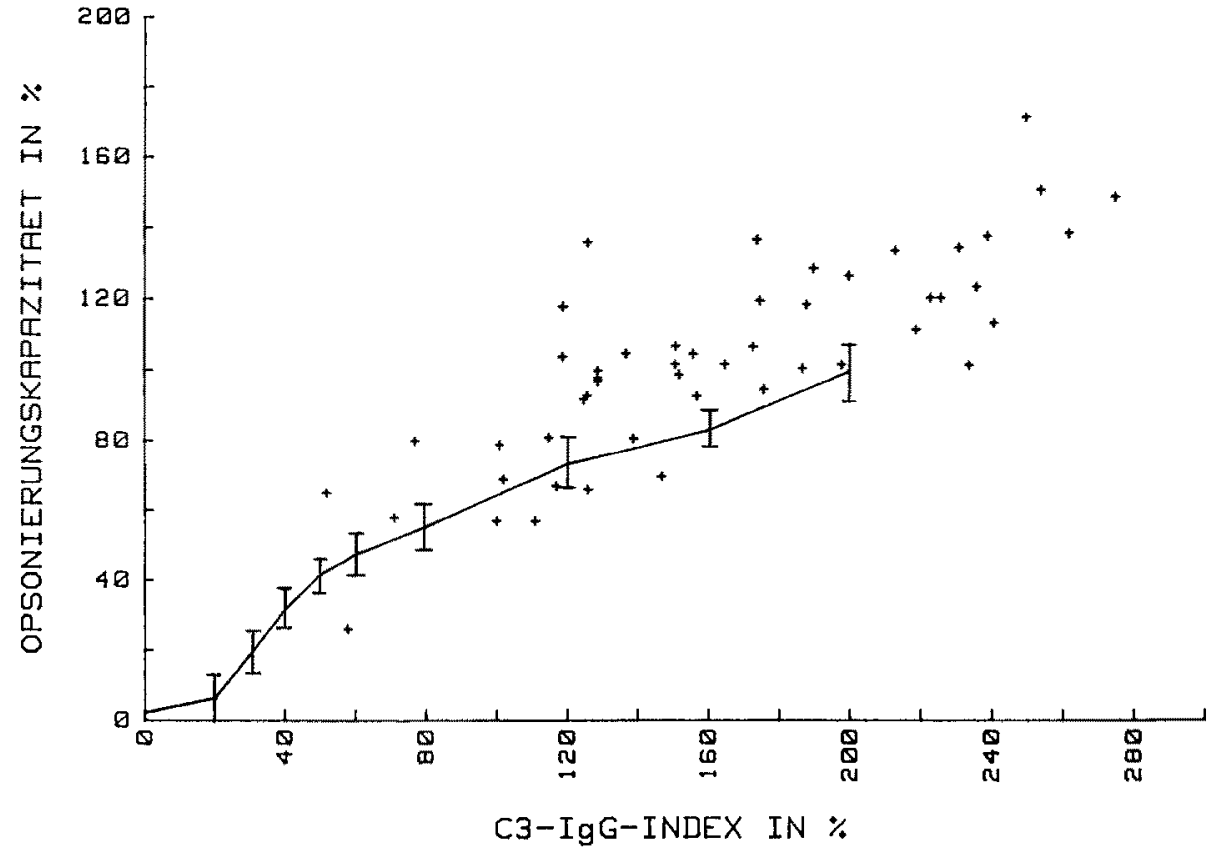

Abb. 2. Opsonierungsverhalten im Patientenserum im Vergleich zu Normalserum. Die Punkte entsprechen den Serumwerten der Peritonitispatienten, die durchgezogene Linie entspricht der Normalserumverdünnungskurve (mit Standardabweichung). Zur Addition des C3 + IgG-Index wurden die jeweiligen Konzentrationen in \% der Serumnorm eingesetzt
Tabelle 1. Opsoninkonzentration $\left(\mathrm{C}_{3}, \mathrm{IgG}\right)$ und Opsonierungskapazität $(\mathrm{OK})$ im Serum von Peritonitispatienten (angegeben sind Mittelwerte und Standardabweichungen

\begin{tabular}{lcc}
\hline Peritonitis & $\begin{array}{l}\text { Akute Peritonitis } \\
(n=13)\end{array}$ & $\begin{array}{l}\text { Persistierende } \\
(n=15)\end{array}$ \\
\hline $\mathrm{IgG}(\mathrm{mg} / \mathrm{d})$ & $785 \pm 366$ & $1364 \pm 385$ \\
$\mathrm{C}_{3}(\mathrm{mg} / \mathrm{dl})$ & $53,6 \pm 18,9$ & $68,1 \pm 17,7$ \\
$\mathrm{OK}(\%$ der Serumnorm $)$ & $85,8 \pm 33,5$ & $115,4 \pm 20,8$ \\
\hline
\end{tabular}

Infektionsherd ist die Passage der entsprechenden Serumfaktoren in die Peritonealhöhle. Die Gesamteiweißkonzentrationen der Exsudate betrugen sowohl bei der akuten Peritonitis als auch während der Etappenlavage zwischen 60-70\% des zugehörigen Serumproteingehaltes. Die Verteilungsmuster der Eiweißfraktionen in den Exsudaten entsprachen weitgehend denen des Serums (Daten nicht dargestellt).

In 28 Peritonitisexsudaten wurden die Konzentrationen des $\operatorname{IgG}$ und $\mathrm{C}_{3}$ immunologisch bestimmt. Die Spiegel lagen - unterschieden nach akuter Peritonitis und persistierender Peritonitis zwischen 31 und $70 \%$ der jeweiligen Serumkonzentrationen (Tabelle 2).

Legte man, wie für das Serum nachgewiesen, einen linearen Zusammenhang zwischen immunologischer $\operatorname{IgG} / \mathrm{C}_{3}-$ Konzentration und der funktionellen Opsonierungskapazität zugrunde, dann war für die im Exsudat gemessenen Opsoninspiegel bei akuter Peritonitis eine durchschnittliche Opsonierungskapazität von $68,4 \%$, bei der Etappenlavage von $67,9 \%$ des Serum-Normalwertes zu erwarten. Unsere Untersuchungen der Opsonierungsleistung der Exsudate mit dem oben genannten modifizierten Chemilumineszenzverfahren ergaben aber wesentlich niedrigere Werte: Die Opsonierungskapazität im Exsudat bei akuter Peritonitis betrug nur $8,4 \%(n=13)$, bei Etappenlavage nur 4,6\% $(n=$ 15). In der Mehrzahl der Exsudate war die Opsonierungskapazität fast vollständig aufgehoben. Nur in wenigen Proben wurden die Sollwerte erreicht, die der in der Serumverdünnungskurve gefundenen Korrelation entsprachen. Bei den Proben mit relativ guter Opsonierungskapazität handelte es sich ausschließlich um klinisch klare bzw. nur gering bakteriell kontaminierte Exsudate. Zum Vergleich wurden 6 sterile Ascitesproben untersucht. Diese zeigten alle eine den Opsoninspiegeln adäquate Opsonierungskapazität.

\section{Ursachen des Opsonierungsdefizits}

Als Ursache der physiologischen Funktionsstörung der Opsonierungsfaktoren im Exsudat ist eine proteolytisch/oxidativ bedingte Inaktivierung der Opsonine bzw. ihr spezifischer Verbrauch anzunehmen. Im Peritonitisexsudat sind PhagozytenEnzyme als Hauptquelle einer proteolytischen Aktivität anzusehen. Als Markerenzym für die lysosomale Degranulation mit extrazellulärer Proteasenfreisetzung wurden in 28 Exsudaten die Spiegel der mit $\alpha_{1}$-Proteinaseinhibitor komplexierten Elastase mittels ELISA bestimmt. Es fanden sich dabei extrem hohe Konzentrationen; mit $250 \mathrm{mg}$ Elastase 
Tabelle 2. Opsoninkonzentration $\left(\mathrm{C}_{3}, \mathrm{IgG}\right)$ und Opsonierungskapazität (OK) im Peritonitisexsudat (angegeben sind Mittelwerte \pm Standardabweichung

\begin{tabular}{lcc}
\hline Peritonitis & $\begin{array}{l}\text { Akute Peritonitis } \\
(n=13)\end{array}$ & $\begin{array}{l}\text { Persistierende } \\
(n=15)\end{array}$ \\
\hline IgG $(\mathrm{mg} / \mathrm{dl})$ & $549 \pm 266$ & $661 \pm 226$ \\
$\mathrm{C}_{3}(\mathrm{mg} / \mathrm{dl})$ & $29,3 \pm 22,8$ & $20,9 \pm 5,6$ \\
OK $(\%$ der Serumnorm) & $8,4 \pm 4,2$ & $4,6 \pm 3,1$ \\
\hline
\end{tabular}

pro Liter wurden Werte bis zum 2000 fachen des Plasma-Normalwertes nachgewiesen (Tabelle 3).

Bei klinisch persistierender Peritonitis konnten wir auch während der Etappenlavage keine Reduktion der Elastasekonzentration im Exsudat feststellen. $\mathrm{Zu}$ Beginn der programmiert alle 2. Tage durchgeführten Relaparotomie waren im Exsudat die ursprünglichen Ausgangskonzentrationen von Gesamteiweiß und Elastase wieder erreicht. Ein Abfall der Elastasespiegel war lediglich zu beobachten, wenn die Peritonitisquelle chirurgisch saniert werden konnte.

Als Marker für die Freisetzung toxischer, oxidativer Granulozytenenzyme wurde die Myeloperoxidase - (MPO) Konzentration im Exsudat gemessen. Es wurden auch hier sehr hohe Konzentrationen, nämlich bis zu $160 \mathrm{mg} / 1$ nachgewiesen (Plasma-Normalwert $36 \mu \mathrm{g} / \mathrm{l}$ ). Im Exsudat bei akuter Peritonitis $(n=13)$ lag die mittlere Konzentration bei $34,5 \pm 42,7 \mathrm{mg} /$, bei persistierender Peritonitis $(n=15)$ betrug die Konzentration $55,4 \pm 39,0 \mathrm{mg} / \mathrm{l}$.
Die Zahl der mittels der Neubauer-KammerTechnik identifizierten Leukozyten im eitrigen Exsudat variierte stark. Die lichtmikroskopisch ermittelte Leukozytenzahl zeigte keine enge Korrelation mit den extrazellulär gemessenen Konzentrationen lysosomaler Enzyme.

Ein möglicher Zusammenhang zwischen der Konzentration proteolytischer Leukozytenenzyme und der Opsonierungskapazität in den untersuchten Exsudaten sollte durch die Korrelation der gemessenen Elastasespiegel mit der jeweiligen Opsonierungsfunktion im Exsudat aufgezeigt werden. Tatsächlich wiesen nur Exsudate mit relativ geringer Elastasekonzentration $(<10 \mathrm{mg} / \mathrm{l})$ eine annähernd normale, d.h. dem Serumverhalten entsprechende Opsonierungsfunktion auf. Exsudate mit einem auffallenden Opsonierungsdefizit waren stärker kontaminiert (Bakterien, Fremdkörper etc.) und enthielten hohe Konzentrationen an komplexierter granulozytärer Elastase (Abb. 3).

Die zweidimensionale Immunelektrophorese mit einem $\mathrm{C}_{3 \mathrm{c}}$-Antikörper erlaubte die Zuordnung der Präzipitationsbanden zu $\mathrm{C}_{3 \mathrm{c}}$-haltigen Proteinfragmenten unterschiedlichen Molekulargewichtes. Wie haben diese Untersuchung im Serum und im Peritonitisexsudat von 6 Patienten durchgeführt. Im Serum der Peritonitispatienten waren mittels der zweidimensionalen Immunelektrophorese $\mathrm{C}_{3}$ Fragmente im Sinne einer $\mathrm{C}_{3}$-Zerstörung nur geringgradig nachweisbar (Abb. 4a). Der überwiegende Anteil des $C_{3}$ lag in seiner kompletten, funktionsfähigen Struktur vor. Als Kontrolle diente das Serum von gesunden Spendern (Abb. 4b). In Peri-

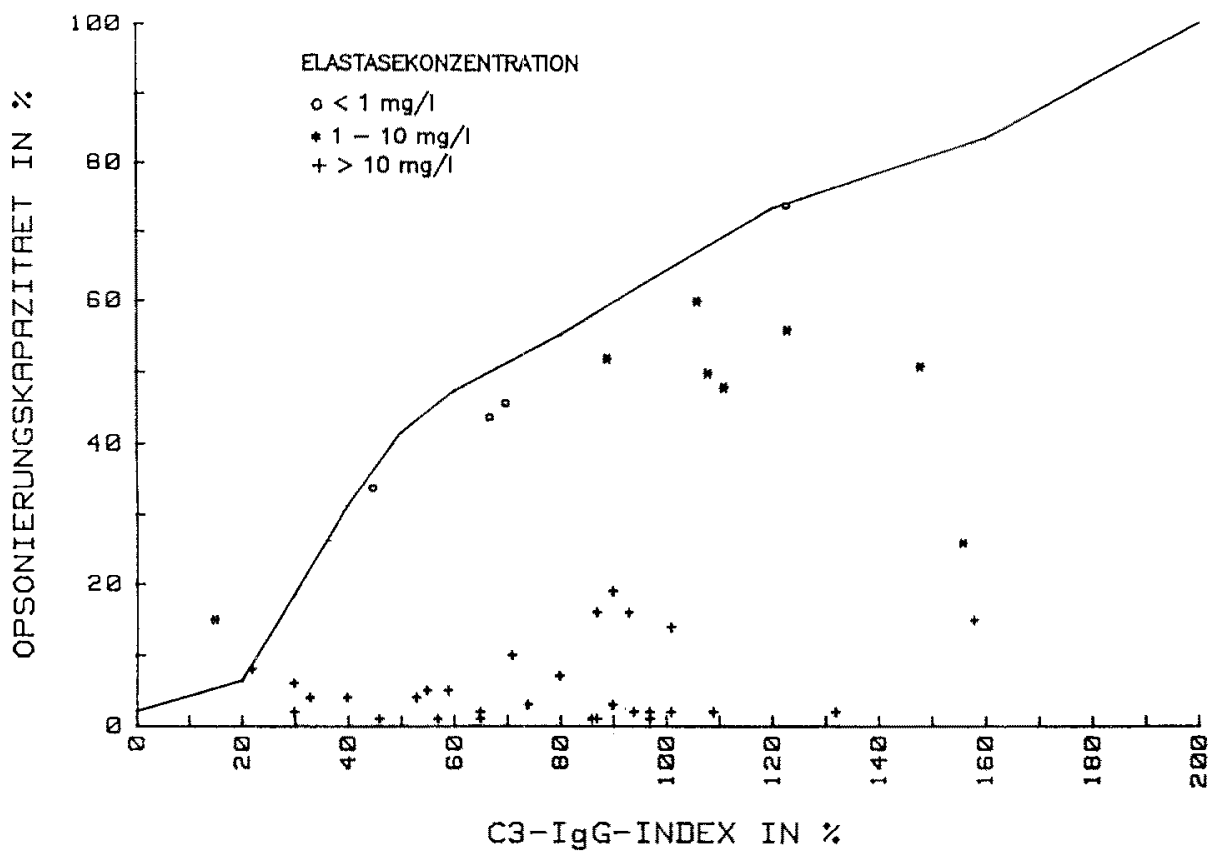

Abb. 3. Abhängigkeit des intraperitonealen Opsonierungsdefizits von der Konzentration der komplexicrten Elastase im Peritonitisexsudat. Die Punkte entsprechen den Exsudatproben, die Kurve zeigt die Korrelation von Opsoninspiegeln und Opsonierungskapazität in der Normalserumverdünnung. Nur Exsudate mit geringem Elastasegehalt weisen eine annähernd adäquate Opsonierungskapazität auf 


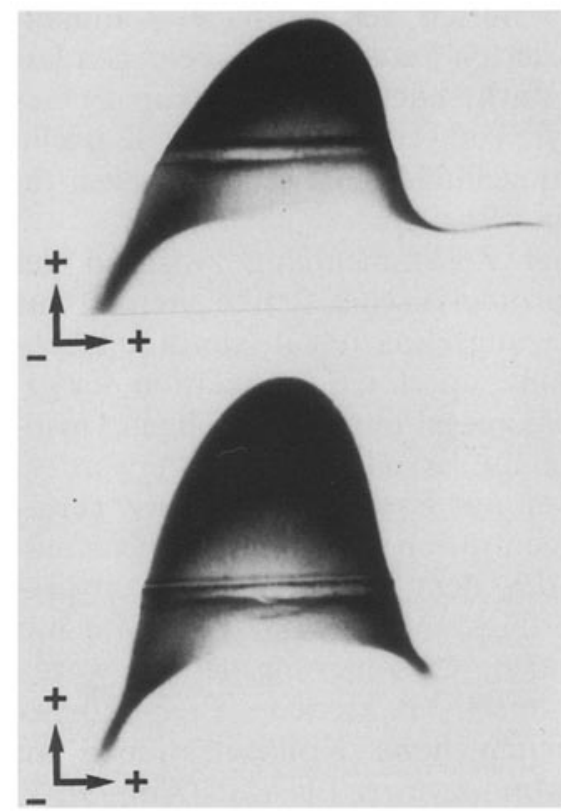

Abb. 4 a, b. Zweidimensionale Immunelektrophorese im Serum von Peritonitispatienten (a), bzw. von Normalprobanden (b) unter Verwendung von $\mathrm{C}_{3 \mathrm{c}}$-Antikörpern. Im Peritonitisserum ist eine geringe $C_{3}$-Spaltung nachweisbar

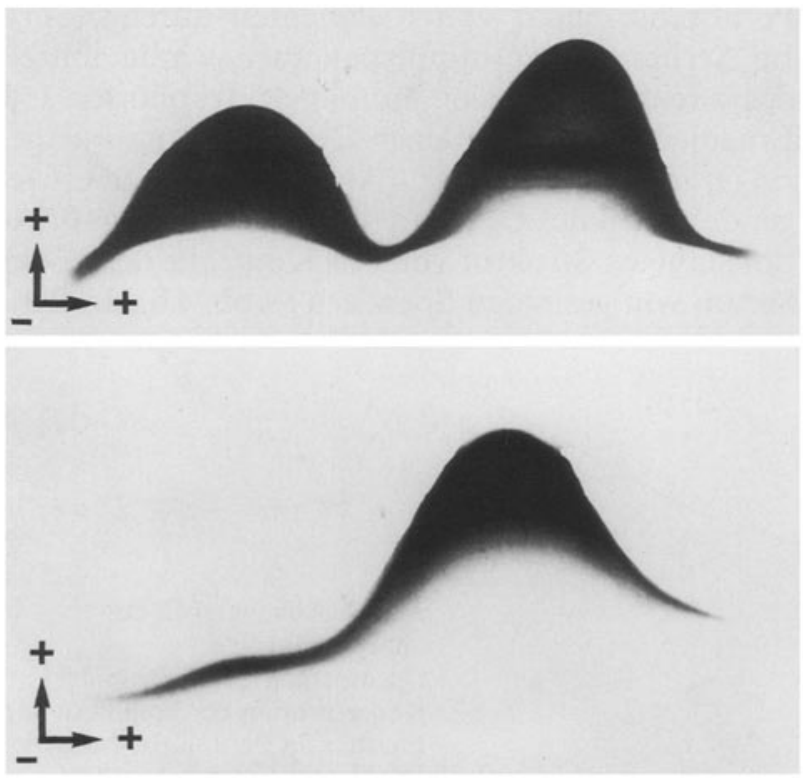

Abb. 5a, b. Zweidimensionale Immunelektrophorese in eitrigem Peritonitisexsudat. a Mäßig eitriges Exsudat (22000 Leukozyten pro $\mathrm{mm}^{3}$ ): Ein erheblicher Teil des $\mathrm{C}_{3}$ ist zu kleineren Bruchstücken gespalten, es ist aber noch intaktes $\mathrm{C}_{3}$ (links) nachweisbar. b Florides Peritonitisexsudat (110000 Leukozyten pro $\mathrm{mm}^{3}$ ): Hochmolekulares, intaktes $C_{3}$ ist kaum mehr nachweisbar

tonitisexsudaten war dagegen der überwiegende Anteil des $\mathrm{C}_{3}$-Pools zu Fragmenten mit niedrigerem Molekulargewicht gespalten, die Präzipitationslinien mit dem $\mathrm{C}_{3 \mathrm{c}}$-Antikörper waren nach
Tabelle 3. Konzentration der komplexierten Granulozyten-Elastase bei Peritonitispatienten (angegeben sind Mittelwerte \pm Standardabweichung, Plasmanormbereich $50-188 \mu \mathrm{g} / 1)$

\begin{tabular}{lcc}
\hline Peritonitis & $\begin{array}{l}\text { Akute Peritonitis } \\
(n=13)\end{array}$ & $\begin{array}{l}\text { Persistierende } \\
(n=15)\end{array}$ \\
\hline Plasma $(\mu \mathrm{g} / 1)$ & $442 \pm 183$ & $248 \pm 55$ \\
Exsudat $(\mu \mathrm{g} / 1)$ & $75972 \pm 52366$ & $89853 \pm 67570$ \\
\hline
\end{tabular}

rechts, d.h. in Richtung höherer elektrophoretischer Mobilität verschoben (Abb. 5a). In Exsudaten mit einem drastischen Opsonierungsdefizit und sehr hohen extrazellulären Konzentrationen an Granulozytenproteinen war intaktes, hochmolekulares $\mathrm{C}_{3}$ mit dieser Methode kaum mehr nachweisbar (Abb. 5b).

\section{Diskussion}

Das von uns modifizierte Chemilumineszenzverfahren stellt eine relativ wenig aufwendige, quantitative Methode für die selektive Bestimmung der Opsonierungskapazität in Serum und Exsudaten dar. Durch die Standardisierung der zellulären Komponente des Meßsystems mit Spenderblut ist die isolierte Untersuchung der humoralen Faktoren des phagozytären Systems möglich. Die gleichzeitige Bestimmung der Opsonierungskapazität von Normalserum als Bezugsgröße in jeder Versuchsreihe garantiert eine gute Vergleichbarkeit der Ergebnisse. Im Patientenserum läßt sich mit diesem Meßsystem die herausragende Bedeutung von IgG und Komplement $\mathrm{C}_{3}$ für die Fremdkörperopsonierung bestätigen. Durch die Verwendung von Vollblut als Phagozytenquelle werden makrophagen-spezifische Opsonine (z.B. Fibronektin) kaum erfaßt (Marino 1988).

Über die Funktion der humoralen Komponente der Infektabwehr im Peritonitisexsudat lagen bisher keine detaillierten Untersuchungen vor.

Unsere Ergebnisse belegten zum ersten Mal einen drastischen Defekt der Fremdkörperopsonierung im menschlichen Peritonitisexsudat. Diese folgenschwere Funktionsstörung beruht offensichtlich nur in geringem Ausmaß auf einer vergleichsweise zum Serum erniedrigten immunologisch meßbaren Opsoninkonzentration. Mit der zweidimensionalen Immunelektrophorese gelang der Nachweis, daß die Opsonine im Exsudat überwiegend als (offensichtlich funktionsunfähige) Bruchstäcke vorliegen. Ähnliche Ergebnisse wurden in infizierten Exsudaten beim Pleuraempyem beobachtet (Waldvogel 1984). Auch dort konnte eine weitgehende Spal- 
tung von $\mathrm{C}_{3}$-Komplement als Ursache eines massiv gestörten Opsonierungssystems nachgewiesen werden.

Die Beeinträchtigung von Opsonierung (z.B. durch proteolytische Spaltung bzw. oxidative Inaktivierung der Opsonine) und Phagozytoseleistung durch lysosomale Phagozytenenzyme ist in vitro nachgewiesen (Coble 1984). Da eine erhebliche extrazelluläre Freisetzung lysosomaler Enzyme bei normaler oder frustraner Phagozytose oder beim Untergang von Leukozyten auch in vivo belegt werden konnte (Duswald 1983; Dwenger 1986; Ohlsson 1977), ist eine entsprechende Wirkung dieser granulozytären Substanzen auf die Opsonierungskapazität verschiedener Körperflüssigkeiten durchaus wahrscheinlich. Im Peritonitisexsudat konnten wir extrem hohe Spiegel für Granulozyten-Elastase und Myeloperoxidase nachweisen.

Derartige Konzentrationen überlasten zumindest lokal das entsprechende Regulationspotential (z.B. $\alpha_{1}$-Proteinaseinhibitor, Antioxidantien). Zusätzlich ist eine oxidative und proteolytische Schädigung der Funktion von Proteinaseinhibitoren wahrscheinlich (Ossana 1986) als deren Folge freie lysosomale Enzymaktivität auftritt. Die proteolytische Degradierung von IgG und Komplement $\mathrm{C}_{3}$ im Pleuraempyemexsudat ist beschrieben (Waldvogel 1984) und daher ebenso für das Peritonitisexsudat anzunehmen.

Wir konnten eine Korrelation zwischen Opsonierungsschaden und extrazellulärer Elastasekonzentration im Peritonitisexsudat feststellen. Ein spezifischer Opsoninverbrauch, z.B. durch physiologische bzw. bakterieninduzierte Aktivierung der Komplementkaskade, aber auch eine proteolytische Zerstörung der Opsonierıngsfaktoren sind daher als wesentliche Ursachen für das nachgewiesene Opsonierungsdefizit im Peritonitisexsudat anzunehmen. Beide Vorgänge führen zu einer Spaltung des hochmolekularen $\mathrm{C}_{3}$-Moleküls in kleinere Fragmente ohne Opsonierungsfunktion. Unter Verwendung eines $\mathrm{C}_{3 \mathrm{c}}$-Antikörpers gelang uns mittels Immunelektrophorese der Beweis, daß im putriden Peritonitisexsudat kaum noch intaktes, d.h. voll funktionsfähiges, aktivierbares $\mathrm{C}_{3}$ vorliegt.

Unsere Ergebnisse konnten erstmals eindeutig zeigen, daß die Störung der körpereigenen intraabdominellen Abwehrfunktion bei akuter Peritonitis aus einem Defekt der humoralen Abwehrkomponente resultiert. Trotz intakter Phagozyten am Peritonitisherd kommt es, bedingt durch ein Versagen der Fremdkörpererkennung und Markierung, zu einem Erliegen der intraabdominellen Abwehrmechanismen, als deren Folge eine Sepsis mit kon- sekutivem Organversagen ohne therapeutische Intervention kaum vermeidbar ist.

Wir danken Fr. Dipl.-Ing. B. Schmidt vom Nephrologischen Forschungslabor der Med. Klinik I der Universität München, Klinikum Großhadern, für die Durchführung der Immunelektrophoresen.

\section{Literatur}

1. Allen RC (1977) Evaluation of serum opsonic capacity by quantitating the initial chemiluminescent response from phagocyting polymorphnuclear leukocytes. Inf and Imm $15: 828-833$

2. Allen RC, Lieberman M (1984) Kinetic analysis of microbe opsonification based on stimulated polymorphnuclear leukocyte oxygenation activity. Inf and Imm $45: 475-482$

3. Bellavite P, Dri P, Della Bianca V, Serra MC (1983) The measurement of superoxide anion production by immunglobulin $\mathrm{G}$ and complement component $\mathrm{C} 3 \mathrm{~b}$. Europ $J$ Clin Invest 13:363-368

4. Billing A, Kortmann H (1986) Nachweis zellulärer und humoraler Abwehrdefekte bei der eitrigen Peritonitis mit einem modifizierten Chemilumineszenzverfahren. Acta chir Austriacae 3:340-341

5. Coble BI, Dahlgren C, Hed J, Stendhal O (1984) Myeloperoxidase reduces the opsonizing activity of immunoglobulin $\mathrm{G}$ and complement component $\mathrm{C} 3 \mathrm{~b}$. Biochem et Biophys Acta 802:501-505

6. van Dijk WC, Verbrugh HA, van Rijswijk R, Vos A, Verhoef J (1982) Neutrophil function, serum opsonic activity, and delayed hypersenstivity in surgical patientes. Surgery $92,1: 21-29$

7. Duswald $\mathrm{KH}$, Ring $\mathrm{J}$, Bachmann Th, Stephan W, Brendel W (1976) Verhalten von IgG, IgA und IgM bei aseptischen und septischen postoperativen Verläufen. Lang Arch Chir Suppl Chir Forum 76:68

8. Duswald KH, Jochum M, Fritz H, Schweiberer L (1983) Diminished in vitro release of granulocyte elastase in septicemia after major surgery. Chir Forum: 217-221

9. Dwenger $A$. Schweitzer G (1986) Bronchoalveolar lavage fluid and plasma proteins, chemiluminescence response and protein contents of polymorphnuclear leukocytes from blood and lavage fluid in traumatized patients. J Clin Chem Clin Biochem 24:73-88

10. Eckert P, Naber M, Barbey-Schneider M (1981) Immunglobuline im Serum und im Peritonealsekret in der frühen postoperativen Phase. Chirurg 52:403-408

11. Freischlag J, Backstrom B, Kelly D, Keehn G, Busuttil BAR (1986) Comparison of blood and peritoneal neutrophil activity in rabbits with and without peritonitis. J of Surg Res 40:145-151

12. Ganroth PO (1972) Crossed immunoelectrophoresis. Scand J Clin Lab Invest 29:39-41

13. Grabner W, Bergner D, Wermuth G (1970) Mikrozonenelektrophorese auf Membranfolien. Ärztl Lab 16:193

14. Hahn H (1984) Mechanismen der körpereigenen Infektabwehr. FAC 3:139-150

15. Inthorn D, Szceponik Th, Mühlbayer D, Jochum M, Redl H (1987) Studies of granulocyte function (chemiluminescence response) in postoperative infection. In: Schlag $G$, Redl H (eds) First Vienna Schock Forum. Part B Progr in Clin and Biol Res 263B: $51-58$

16. Marino JA, Spragnuolo PJ (1988) Fibronectin and phagocytic clearance mechanisms. J Lab Clin Med 112:493-494

17. Neumann S, Gunzer G, Hennrich N, Lang H (1984) PMN- 
elastase assay: Enzyme immunoassay for human polymorphnuclear elastase complexed with $\alpha_{1}$-proteinase inhibitor. J Clin Chem Clin Biochem 22:693-697

18. Neumann S, Gunzer G, Lang H, Jochum M, Fritz H (1986) Quantitation of myeloperoxidase from human granulocytes as an inflammation marker by enzyme-linked immunosorbent assay. Fresenius $Z$ Anal Chem 324:365

19. Ohlsson K. Olsson I (1977) The extracellular release of granulocyte collagenase and elastase during phagocytosis and inflammatory processes. Scand J Haematol 19:145-152

20. Ossana PJ, Test ST, Matheson NR, Regiani S, Weiss SJ (1986) Oxidative regulation of neutrophil elastase-alpha-1proteinase inhibitor interactions. $\mathbf{J}$ Clin Invest 77:1939-1951

21. Stübner G (1983) Immunologische Aspekte intraperitonealer Infektionen. FAC, Band 2-3:437-445

22. Teichmann H, Wittmann D, Andreone P (1986) Scheduled reoperations (Etappenlavage) for diffuse peritonitis. Arch Surg $121: 147-152$
23. Waldvogel FA, Vaudaux P, Lew PD, Zwahlen A, Suter S, Nydegger U (1984) Deficient phagocytosis secondary to breakdown of opsonic factors in infected exudates. Adv Exp Med and Biol 141:603-610

24. Weichselbaum TE (1946) An accurate and rapid method for determination of proteins in small amounts of blood serum and plasma. Am J Clin Path 16:40

Eingegangen: 18. März 1988

Zurück zur Überarbeitung: 4. November 1988

Angenommen: 4. Januar 1989

Dr. A. Billing

Chirurgische Klinik und Poliklinik

der Ludwig-Maximilians-Universität München

Klinikum Großhadern

Marchioninistr. 15

D-8000 München 70 